\title{
Design of an Energy Detector for Heartbeat Localization in ECG signals
}

\author{
Z. Benjelloun, R. Vauche, W. Rahajandraibe, R. Bouchakour
}

Aix Marseille Univ, Univ Toulon, CNRS, IM2NP, Marseille, France

\begin{abstract}
Cardiovascular Diseases (CVD) causes as many deaths as cancer in Europe. A method to detect them consists in tracking the heartbeat rate from ECG signal. In this case, QRS complexes have to be localized and it is proposed here to use an energy detector to allow this. It can be integrated in an analog circuits and allows low power and low cost applications to be addressed such as consumer embedded electronics. Using $0.35 \mu \mathrm{m}$ CMOS technology from AMS, the design of the analog multiplier cell required by the energy detector is also presented here. It shows a voltage gain upper than 18 with enough linearity for the targeted applications. Preliminary results of the full energy detector which mix Eldo and Matlab simulations show an accuracy upper than $99 \%$ for SNR upper than 0dB.
\end{abstract}

Index Terms - Electrocardiography, Energy Detector, Heart Rate Variability, Multiplier, QRS detection.

\section{INTRODUCTION}

The Heart Rate Variability (HRV) represents the variation of the time intervals between each heartbeat. HRV is normally low for healthy people and increases with the risk of cardiac events such as fibrillation, arrhythmia, and Premature Ventricular Contraction (PVC) equally known as ventricular extrasystole. To know the moments when a heartbeat occurs, QRS complexes can be extracted from an Electrocardiogram (ECG) recording. These QRS complexes, synchronous with heartbeats and which correspond to the depolarization of the right and left ventricles of the human heart, are generally the most visible pulses in a clean ECG signal between P-waves and T-waves as indicated in Fig. 1.

The time intervals between QRS complexes, and also heartbeats, are known in medical papers as RR signal when they are plotted in time domain. Then, to estimate correctly HRV, it is necessary to know this RR signal with the best possible accuracy which is only possible when all QRS complexes are detected [1]. However, ECG recordings may contain distorted episodes due to various extrinsic sources of interferences such as electrical activity produced by skeletal muscles, power-line, motion or electrode artifacts, etc. Moreover, as indicated in [2], other intrinsic reasons can break QRS detection such as wide PVCs which occur before regular heartbeats, high-amplitude of T-waves, low-amplitude QRS complexes, negative QRS complexes, low Signal-to-Noise Ratio (SNR), etc.

To detect QRS complexes as well as possible, several algorithms have been designed. A comparative study of these algorithms has been done in [1] and shows that firstderivative-based methods used in real-time analysis have the best accuracy. The most popular of these QRS detection methods is the Hamilton-Tompkins algorithm [3], which is an improvement of the Pan and Tompkins algorithm proposed in [4]. This algorithm uses a specific adaptive threshold after filtering, differentiation, squaring and integration of the ECG signal. Other algorithms exist as the ones based on Hilbert transform. An example of this type of algorithm is proposed in [5] and uses a threshold which does not need to be experimentally fixed. Finally, Discrete Wavelet Transform (DWT) can be used for QRS detection as proposed in [6] and is based on a multi-resolution sup-band decomposition process and an adaptive threshold depending on the output signal of the moving window integrator.

All the methods mentioned previously are digital solutions which are easily implementable on a microcontroller or digital signal processor. However, even if the used algorithm can be easily changed in this type of systems, they integrate lots of functions which consume power and silicon area. Thus, to address low cost and low power applications such as consumer embedded electronics, it is proposed here to design an analog Application Specific Integrated Circuit (ASIC) dedicated to the QRS complexes detection. Moreover, since QRS complexes are similar to the pulses used in IR-UWB in terms of waveforms, a solution inspired from previous works on non-coherent Ultra-Wide-Band Impulse Radio (IR-UWB) receivers [7] is proposed here.

In IR-UWB, it is possible to find in literature two families of non-coherent receivers which are known for their simplicity; the first is based on peak voltage detection and the second one on energy detection. In [7], a non-coherent IRUWB receiver based on energy detection is presented. It consists in a Low Noise Amplifier (LNA), a squarer, an envelope detector and a decision stage. This structure is here the starting point of the presented study and the aim of this paper is also to provide the necessary elements to transform this UWB pulse detector to a QRS detector, and to provide a squarer compatible with QRS detection.

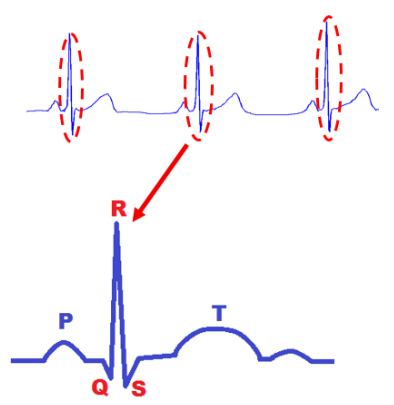

Fig. 1. Waveform of a QRS complex. 
In this paper, to adapt the structure presented in [7] to QRS complexes detection, a preliminary study on QRS spectrum is done in Section II before the sizing of the detection chain. Next, Section III focuses on the design of the squarer which is the key element of the proposed QRS detector since it has to work on a sufficient input voltage range and consumes as few as possible. Section IV presents the first simulations results of the full chain where Eldo simulation results of the squarer have been mixed to Matlab computing. Finally, conclusions and prospects are presented in Section V.

\section{ARCHITECTURE OF THE QRS COMPLEXES DETECTOR}

As seen previously, heartbeat rate can be obtained by computing the time interval between two consecutive QRS complexes. An efficient real time processing is also needed to detect them even if the ECG signal is distorted. As low power and low cost applications are targeted, the processing must be achievable in an analog ASIC and it is proposed here to adapt the IR-UWB pulse receiver presented in [7]. For these reasons, QRS complexes are characterized in frequency domain which allows the processing chain to be sized.

\section{Domain}

\section{A. Characterization of QRS Complexes in Frequency}

The relative power spectrum averaged on 150 beats of QRS complexes and its interference sources are provided in [8] and shown in Fig. 2. It is possible to see that the central frequency of QRS complex is about $10 \mathrm{~Hz}$ and its $-10 \mathrm{~dB}$ bandwidth to $10 \mathrm{~Hz}$. To confirm that, the distribution of the central frequency of QRS complexes has been studied with the help of the public ECG database Physionet. Results are given in Fig. 3 and shows that the central frequency is uniformly spread in the $8-12 \mathrm{~Hz}$ range. It is also confirmed that the typical central frequency of QRS complexes is $10 \mathrm{~Hz}$.

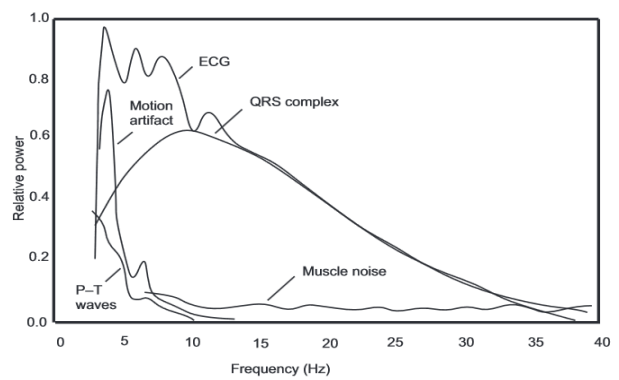

Fig. 2. Relative power spectra of QRS complex, $\mathrm{P}$ and $\mathrm{T}$ waves, muscle noise and motion artifacts based on an average of 150 beats [8]

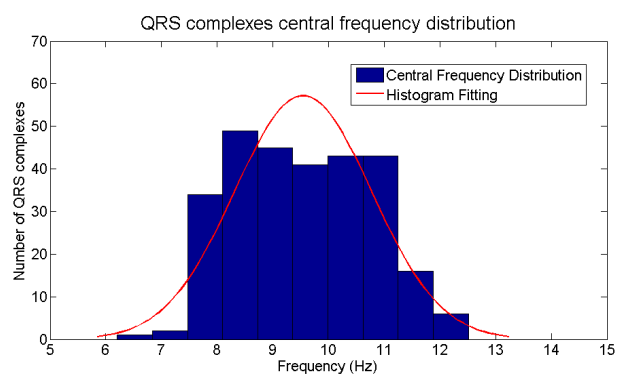

Fig. 3. Central frequency distribution of 286 QRS complexes (150 QRS complexes are from MIT-NSR and 136 from Apnea Physionet database).

\section{B. QRS detectors processing chain}

QRS detection algorithm typically includes four signal processing steps: (i) a bandpass filter, (ii) a nonlinear transformation, (iii) a moving window integrator and (iv) a decision rule algorithm. The architecture of the IR-UWB noncoherent receiver based on energy detection presented in [7] has a similar approach where, the non-linear transformation is a squaring function and the moving window integrator is replaced by a low-pass filter (which allows envelope of squared pulses to be extracted too). As QRS complexes and IR-UWB pulses have closed waveforms, the QRS detection chain based on energy detection proposed here consists in a bandpass filter (a), a squarer (b), an integrator (c) and an adaptive threshold comparator (d) as shown in Fig. 4. The Matlab simulation results of this structure are given in Fig. 5.

The choice of characteristics of the bandpass filter (a) is a critical step in the processing chain design since it should eradicate the noise interfering with the targeted signal without filtering it. From results of the previous study, it has been chosen with a $-10 \mathrm{~dB}$ bandwidth of $8 \mathrm{~Hz}$ centered on $10 \mathrm{~Hz}$. It allows then the baseline (DC block) and the main $(50 \mathrm{~Hz}$ or $60 \mathrm{~Hz}$ ) to be filtered. Moreover, noise from skeletal muscles, $\mathrm{T}$-waves and motion artifacts are reduced too since their power spectrum is concentrated below $5 \mathrm{~Hz}$ as shown in Fig. 2. Finally, as this stage works as an LNA, it should have a low noise figure especially to minimize $1 /$ f noise and a sufficient gain to get a peak signal arbitrary fixed to $100 \mathrm{mV}$ to obtain usable signals after squaring operation.

As the proposed QRS complexes detector is based on an energy detector, the second block is a squarer (b). This allows to stronger signals to be more amplified than weak signal. However, although this operator is easy to implement in a digital system, it is more difficult to integrate in an analog ASIC when a large signal has to be squared. For these reasons, the design of a low power and low cost multiplier is proposed in the following section.

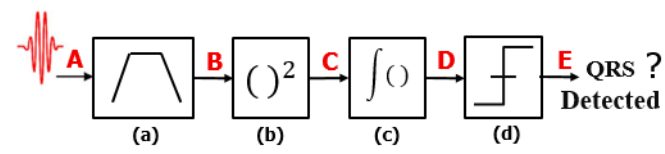

Fig. 4. Block diagram of the processing chain.

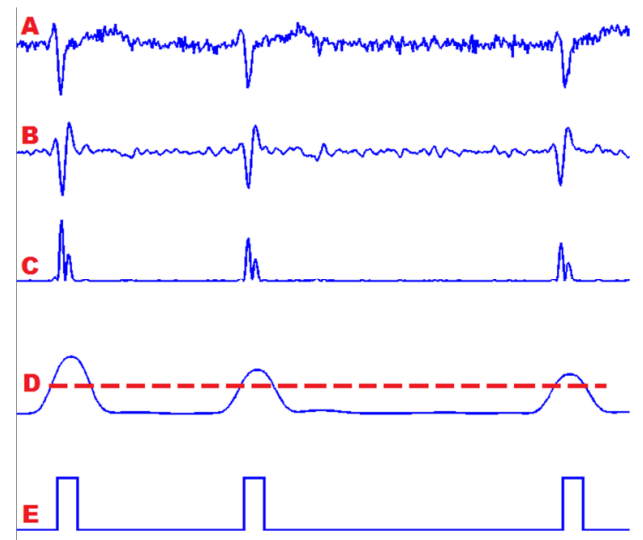

Fig. 5. Illustration of the different processing steps. 
It is now necessary to extract envelope of the squared signal which is done here by a sliding window integrator (c). The key parameter to adjust in this block is the window width. If the window is too wide, two QRS complexes can be interpreted as only one and if the window is too narrow, there is a risk of missing the QRS peaks. From experiments, the width has been chosen equal to $150 \mathrm{~ms}$ which correspond to the double of the founded average QRS width.

Finally, an adaptive threshold comparator (d) has to be used to keep only the larger pulses normally due to QRS complexes. This threshold is fixed equal to $60 \%$ of the maximum value of the input signal during the last $5 \mathrm{~s}$ as proposed in [8]. Moreover, to filter pulses due to interferences, this adaptive threshold comparator is not retriggerable during $366 \mathrm{~ms}$ since the maximum heartbeat is equal to $220 \mathrm{bpm}$ for a human. To improve accuracy of this block, a QRS complex is considered detected when its input is higher than the threshold, and when its first derivative is equal to 0 which indicates a local maximum.

\section{SQUARER DESIGN}

In the case of an analog ASIC, a squaring stage can be obtained by using an analog voltage multiplier. This multiplier has to perform a linear products between its two input voltages $\left(v_{1}, v_{2}\right)$, and also provides on its output a voltage which can be expressed as $\mathrm{K} . \mathrm{v}_{1} \cdot \mathrm{v}_{2}$, where $K$ is a constant gain factor. The performances evaluation of this multiplier has also to include linearity, power consumption, and especially input range and gain. This is due to the fact that stronger signals are be more amplified than weak signal. In this section, a differential multiplier is designed and integrated in an unbalanced squarer using a $0.35 \mu \mathrm{m}$ CMOS design kit from AMS.

\section{A. Differential Multiplier Design}

In [10], a comparison between different CMOS transconductance multipliers structures is done. It appears that they can be categorized into two groups below MOS operation region: linear and saturation region. In this paper, the designed multiplier operates in saturation region in order to obtain sufficient input range and gain according to applications requirements. The proposed design shown in Fig. 6 is based on a Gilbert mixer with balanced inputs (V1 and V2) and unbalanced output (VOP). To drive correctly the multiplier, balanced inputs $\mathrm{VxP}$ and $\mathrm{VxN}$ have to be symmetrical with respect to VDD/2. Finally, it can be turned-off when it is necessary by disabling the current source since PDB is a logic signal equal to ' 0 ' (GND) or ' 1 ' (VDD).

\section{B. Integration in an Unbalanced Squarer}

To drive the proposed multiplier with an unbalanced filtered ECG signal, it is necessary to generate symmetrical inputs signals with respect to VDD/2. A solution based on operational amplifier is proposed in Fig. 7 and allows symmetrical filtered ECG signals to be obtained with respect to VOFFSET. It consists in a voltage follower connected to two substractors, the first being used to provide high impedance to ECG source since the equivalent input impedance of the both connected substractors is R. To limit the power consumption of operational amplifiers and make a compromise with silicon area, $\mathrm{R}$ has been fixed to $100 \mathrm{k} \Omega$.

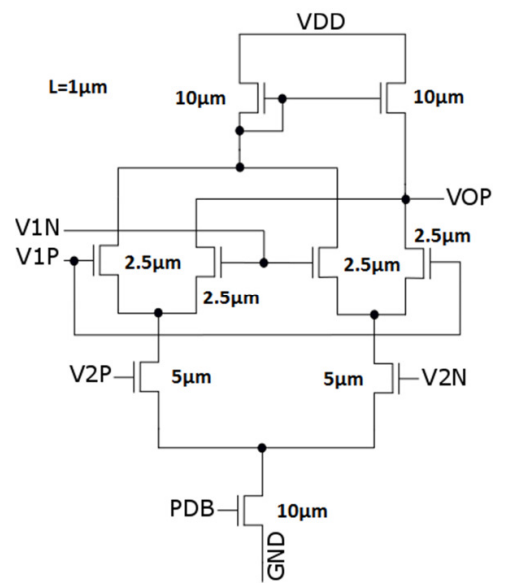

Fig. 6. Design of the presented balanced multiplier.

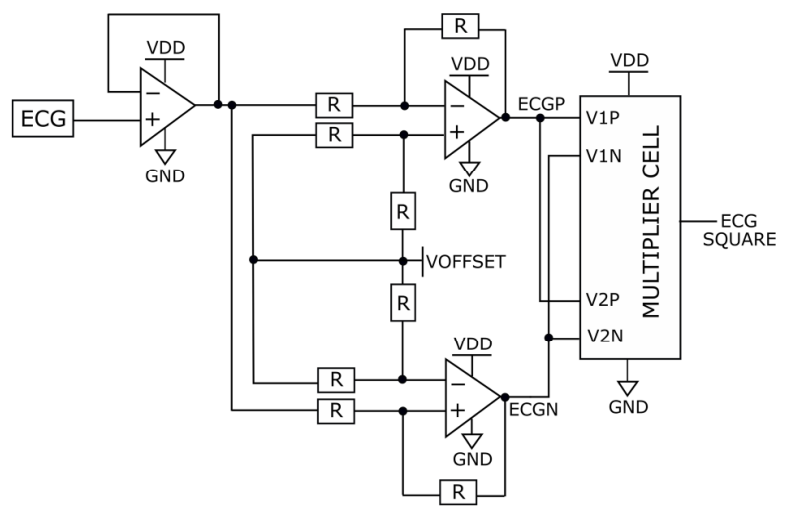

Fig. 7. Integration of the balanced multiplier in a unbalanced squarer.

\section{Simulation Results}

The presented energy detector for heartbeat localization in ECG signals has been post-layout simulated using a $0.35 \mu \mathrm{m}$ CMOS design kit from AMS with a supply voltage of $3.3 \mathrm{~V}$. The operational amplifiers required by the squarer are taken from AMS IP library. The layouts of the designed multiplier and of the full squarer are shown in Fig. 8 and respectively have a core area of $20 \mu \mathrm{m}$ by $31 \mu \mathrm{m}$ and $1 \mathrm{~mm}$ by $0.9 \mathrm{~mm}$. The following part gives multiplier characteristics. Next, examples of energy detector output signal obtained by Cadence and Matlab mixed simulations are provided.

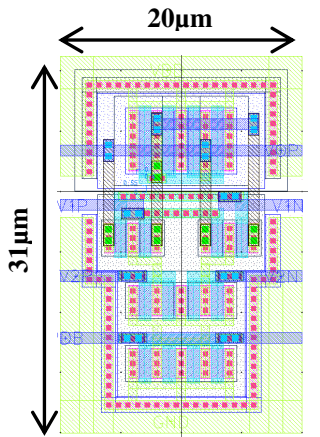

(a)

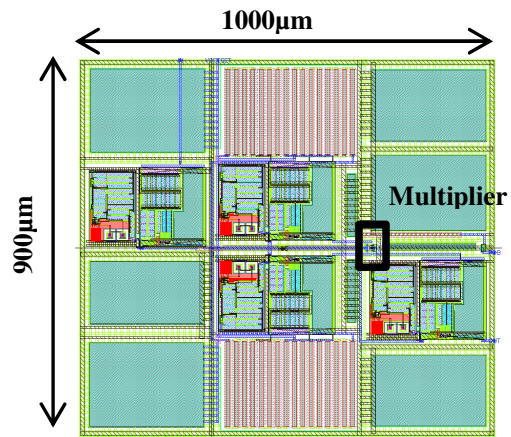

(b)
Fig. 8. Layout of the multiplier (a) and of the full squarer (b). 


\section{A. Multiplier characterization}

To characterize the multiplier, the common mode voltage of $\mathrm{V} 1$ and $\mathrm{V} 2$ which is equal to $(\mathrm{VxP}+\mathrm{VxN}) / 2$ is set to $\mathrm{VDD} / 2$ $(1.65 \mathrm{~V})$. The differential mode voltage of V1 (resp. V2) which is equal to $(\mathrm{VxP}-\mathrm{VxN}) / 2$ is called $\mathrm{v} 1$ (resp. v2). The results of the multiplier DC analysis for an input range of $[-200 \mathrm{mV}$; $200 \mathrm{mV}]$ is given in Fig. 9. It appears that (VOP - VDD/2) can be identified to K.v1.v2 where $\mathrm{K}$ is equal to 18.3 (resp. 18.5) with a standard derivation of 0.67 (resp. 1.03) for $[-100 \mathrm{mV}$; $100 \mathrm{mV}]$ (resp. [-200mV; 200mV]) vx voltage range. Finally, its power consumption is $0.94 \mathrm{~mW}$ when it is turned-on (PDB $=\mathrm{VDD})$ and about $17 \mathrm{pW}$ when it is turned-off $(\mathrm{PDB}=\mathrm{GND})$.

\section{B. Squarer simulation}

To simulate the presented energy detector in the worst case, an ECG signal containing many irregularities from Physionet database has been used. The blocks (a), (c) and (d) which can be seen on Fig. 4 have been simulated with Matlab and the block (b) has been post-layout simulated with Eldo. The gain of the bandpass filter (a) has been tuned to have a maximum peak voltage of $100 \mathrm{mV}$ on its output. Simulation results of the processing chain are shown on Fig. 10. They show the filtered ECG voltage $B$ applied to the squarer and the voltage C obtained before the integration stage, the both for an infinite (a) and OdB (b) SNR using an Additive White Gaussian Noise (AWGN) to the ECG input voltage. Finally, simulations of the full energy detector show that 99.2\% (resp. 100\%) of QRS peaks are well detected for a $0 \mathrm{~dB}$ (resp. > 3dB) SNR.

\section{CONCLUSION}

To extract heartbeat rate from ECG signal, a QRS detector has been proposed. It is based on energy detection and requires also a squarer function. To implement it in a low cost and low power analog ASIC, an analog multiplier cell has been designed and post-layout simulated with Eldo. It shows a voltage gain upper than 18 with enough linearity. To estimate performances of the full processing chain, the other required functions have been simulated with Matlab. Preliminary results which mix Eldo and Matlab simulations show a good accuracy for SNR upper than $0 \mathrm{~dB}$, which allows the use of the presented QRS detector to be expected for cardiac monitoring [11] or other physiological parameters by modifying characteristics (frequency band, etc.) of the energy detector.

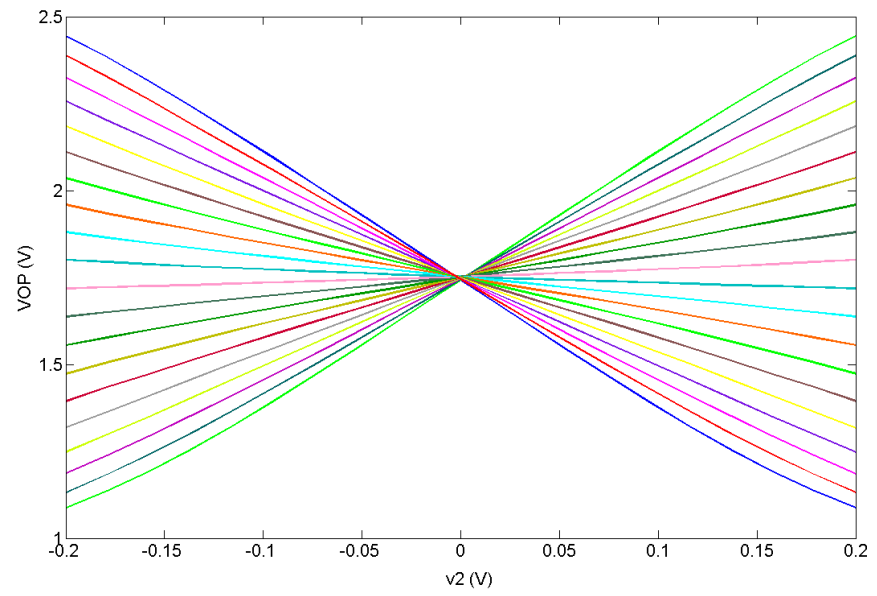

Fig. 9. DC analysis of the multiplier: VOP vs (v1, v2).
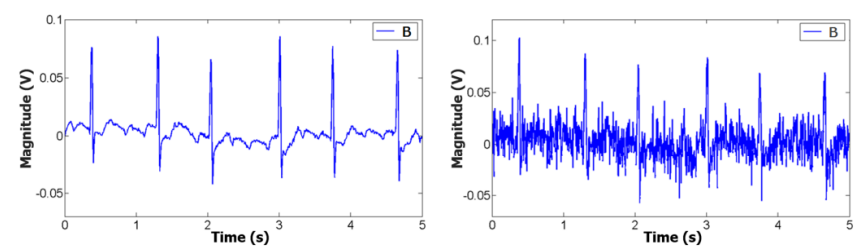

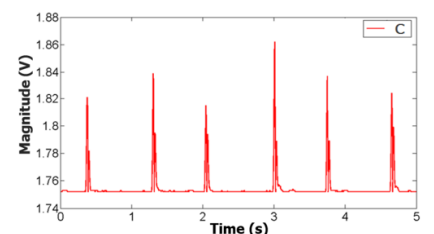

(a)

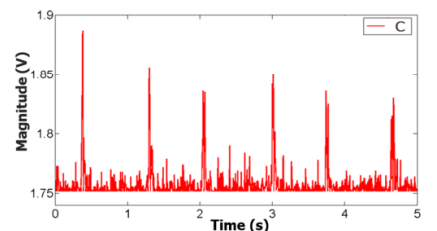

(b)
Fig. 10. Simulations results of the filtered ECG voltage B applied to the squarer and the voltage $\mathrm{C}$ obtained before the integration stage for an infinite (a) and OdB (b) SNR.

\section{ACKNOWLEDGMENT}

This work, which is part of the APPRISE/HIT project, was performed with the support of the A*MIDEX project $\left(\mathrm{n}^{\circ}\right.$ ANR-11-IDEX-0001-02) funded by the «Investissements d'Avenir» program of the French Government, which is managed by the French National Research Agency (ANR).

\section{REFERENCES}

[1] G. G. Bernston and J. R. Stowell, "ECG artifacts and heart period variability: Don't miss a beat! " Department of Psychology and Neuroscience Program, The Ohio State University, Columbus, USA. May 7, 1997

[2] N. M. Arzeno, Z. D. Deng and C. S. Poon, "Analysis of First-Derivative Based QRS Detection Algorithms," in IEEE Transactions on Biomedical Engineering, vol. 55, no. 2, pp. 478-484, Feb. 2008.

[3] P. S. Hamilton and W. J. Tompkins, "Quantitative Investigation of QRS Detection Rules Using the MIT/BIH Arrhythmia Database," in IEEE Transactions on Biomedical Engineering, vol. BME-33, no. 12, pp. 1157-1165, Dec. 1986.

[4] J. Pan and W. J. Tompkins, "A Real-Time QRS Detection Algorithm," in IEEE Transactions on Biomedical Engineering, vol. BME-32, no. 3, pp. 230-236, March 1985.

[5] D. S. Benitez, P. A. Gaydecki, A. Zaidi and A. P. Fitzpatrick, "A new QRS detection algorithm based on the Hilbert transform," Computers in Cardiology 2000, Cambridge, MA, pp. 379-382, 2000.

[6] S. M. Szilagyi and L. Szilagyi, "Wavelet transform and neural-networkbased adaptive filtering for QRS detection," Engineering in Medicine and Biology Society, 2000. Proceedings of the 22nd Annual International Conference of the IEEE, Chicago, IL, vol. 2, pp. 12671270, 2000.

[7] O. Ramos Sparrow, R. Vauché, N. Dehaese, S. Bourdel, J. Gaubert, I. Ben amor, E. Muhr, P. Losco, O. Fourquin, "High rate UWB CMOS transceiver chipset for WBAN and biomedical applications," Analog Integrated Circuits and Signal Processing, vol. 81, no. 1, pp. 215-227, 2014.

[8] Valtino X. Afonso. 1993. ECG QRS detection. In Biomedical digital signal processing, Willis J. Tompkins (Ed.). Prentice-Hall, Inc., Upper Saddle River, NJ, USA 236-264.

[9] P. Z. Peebles, probability random variables and random signal principles New-York: McGraw-Hill, ch 9, pp. 577-584, 1980.

[10] Gunhee Han and E. Sanchez-Sinencio, "CMOS transconductance multipliers: a tutorial," in IEEE Transactions on Circuits and Systems II: Analog and Digital Signal Processing, vol. 45, no. 12, pp. 1550-1563, 1998.

[11] W.D. Lynn, O.J. Escalona, D.J. McEneaney. "Arm and writ surface potential mapping for wearable ECG rhythm recording devices: a pilot clinical study," Journal of Physics: Conference Series, vol. 450, no. 1, 2013. 